\title{
Extending Character-based Storytelling with Awareness and Feelings
}

\author{
David Pizzi, Marc Cavazza and Jean-Luc Lugrin \\ University of Teesside \\ Middlesbrough, TS1 3BA \\ United Kingdom \\ +44 1642342657 (650) \\ \{d.pizzi, m.o.cavazza, j-I.lugrin\} @tees.ac.uk
}

\begin{abstract}
Most Interactive Storytelling systems developed to date have followed a task-based approach to story representation, using planning techniques to drive the story by generating a sequence of actions, which essentially "solve" the task to which the story is equated. One major limitation of this approach has been that it fails to incorporate characters' psychology, and as a consequence important aesthetic aspects of the narrative cannot be easily captured by Interactive Storytelling. In this paper, we introduce a new approach to Interactive Storytelling, which aims at reconciling narrative actions with the characters' attributed psychology as stated in the narrative. Our long-term goal is to be able to explore Interactive Storytelling for those narrative genres which are based on the characters' psychology rather than solely on their actions. We used as a starting point the formalisation by Flaubert himself of his novel Madame Bovary, which includes a detailed account of characters' desires and feelings. We describe a prototype in which characters' behaviour is driven by a real-time search-based planning system applying operators whose content is based on a specific inventory of feelings. Furthermore, the actual pattern of evolution of the character's plan, as measured through the variation of the search heuristic, is used to confer a sense of awareness to the characters, which can be used to generate feelings about its overall situation, from feelings of boredom to hope.
\end{abstract}

\section{Categories and Subject Descriptors}

I.2.1 [Artificial Intelligence]: Games

\section{General Terms}

Theory, Design, Algorithm, Experimentation, Verification

Permission to make digital or hard copies of all or part of this work for personal or classroom use is granted without fee provided that copies are not made or distributed for profit or commercial advantage and that copies bear this notice and the full citation on the first page. To copy otherwise, to republish, to post on servers or to redistribute to lists, requires prior specific permission and/or a fee.

AAMAS'07 May 14-18 2007, Honolulu, Hawai'i, USA.

Copyright 2007 IFAAMAS.

\section{Keywords}

Embodied and Believable Agents, Interactive Storytelling, Cognitive Models

\section{INTRODUCTION}

One of the main challenges of Interactive Storytelling (IS) is to determine the real-time behaviour of virtual characters in a way that is consistent with narrative phenomena. The duality between character and plot emphasises the difficulty of reconciling a character's perspective with the control exerted by the storyline. In addition, the psychology and feelings of characters is an essential part of most dramas. It is therefore some kind of a paradox to see these under-represented in interactive narrative research, to the exception of simulation and educational storytelling [13] [1]. One possible explanation is that the emphasis on narrative representations has essentially been on narrative actions rather than characters' psychology, feelings and motivations. Characters' psychology has been diluted into mythical narrative functions, such as treason, which were supposed to subsume the psychological determinants of characters' actions. Throughout the narrative genres that have served to illustrate research in interactive narrative, there has been a prevalence of those genres based on actions and situations rather than the psychological relations between agents. One notable exception has been Mateas and Stern's Façade system [14]. One of the central aspects of Façade, which consists in departing from the "task completion" approach to interactive storytelling to investigate the aesthetic satisfaction of the narrative, is certainly the best description of the problems facing those investigating IS from the perspective of new media and digital entertainment.

\section{PLANNING AND THE NARRATIVE DRIVE}

The majority of Interactive Storytelling research prototypes have made use of planning for their baseline narrative engine, generating action sequences as the story backbone [15] [4] [11]. In our own previous work [4] [5] we have developed an approach known as character-based storytelling, to the extent that characters' roles, rather than a centralised plot model, serve as the main driver for narrative generation. From a technical perspective these roles display an internal structure, which is also task-based, with an implicit level of intentions predicated on the story structure. This approach is suited to the generation of situations arising from the interaction between characters. We wanted to 
explore another approach, which consists in adopting a weaker representational model, in which planning is only used as a technology driving action selection towards certain long-term objectives. In other words, a character can be driven by long-term motivations such as wealth, happiness or fame rather than by a fine-grained goal description in terms of state of affairs. These motivations can be formalised as conjunction of feelings constituting the "goal state" for the planning system. Our planning component is a standard HSP-type planner [2] controlling STRIPS-like operators [6]. The planner has to operate in a dynamic environment because of the interactions between characters (they all interact with each other and share the same physical space) and the potential for user intervention. In order to adapt it to real-time planning we have used RTA* [9] as its underlying search algorithm. Our implementation generates the next best action to be carried out by a character, without using a horizon-bound look-ahead step.

\section{NARRATIVE FORMALISATION}

As we want to depart from the task-oriented model of a narrative, the representation of the goal state for the HSP planner should not correspond to a specific final state of affairs described in terms of situational predicates. The goal state should thus be mainly composed of feelings and, most importantly, will essentially behave as a driver rather than a specific goal to be reached. Another important aspect is that these driving goals should contain a dynamic element, i.e. they are updated as the situation evolves, some desires being added and others deleted.

In our HSP planner, each state is defined as a conjunction of character's feelings and states of mind (e.g. Adultery-AcceptedRisk(Emma) ) and state of affairs in the physical world (e.g. Outside(Emma, House) ). Operators are divided into two categories. Interpretation Operators are actually in charge of a specific form of appraisal and they update the characters' feelings as a function of the current situation (previous feelings affected by recent evolutions in state of affairs). Action Operators correspond to physical actions (such as changing location) including communication between characters (e.g. Emma declaring her love to Rodolphe).

\section{AWARENESS AND FEELINGS}

Early models of inclusions of emotions in planning have been based on appraisal theory [7] [8] [13], i.e. emotions were related to an evaluation by the agent of its own situation, either with respect to its desires, or considering its social relations to other agents (including status and responsibility). Very often, anticipation of negative outcomes or threats to its plan has been the main drive for emotion generation. Anticipation is one particular case of the awareness on the character's side of the evolution of its situation. The other aspect often illustrating emotional planning is coping [1] [12]. In line with our commitment to the exploration of aesthetic phenomena in the narrative, we are looking for a representation that would not deduce character's psychology from first (cognitive) principles. Mechanisms of appraisal and coping could also be described for Emma Bovary, but a more appropriate level of description would make the best use of those specific semantic domains in relation to the story topics, using the more specific feelings described by Flaubert [10]. In other words we want to explore a narrative rather than a cognitive approach. If we want to relate the character's psychology to aesthetic elements of the narrative, we need to find a model in which this global awareness can also be related to narrative descriptions. In modern narratology, Bremond [3] has proposed to describe story progression through the evolution of characters' situation in terms of improvement and deterioration, including multiple causal descriptions of the other characters' influence on the process.

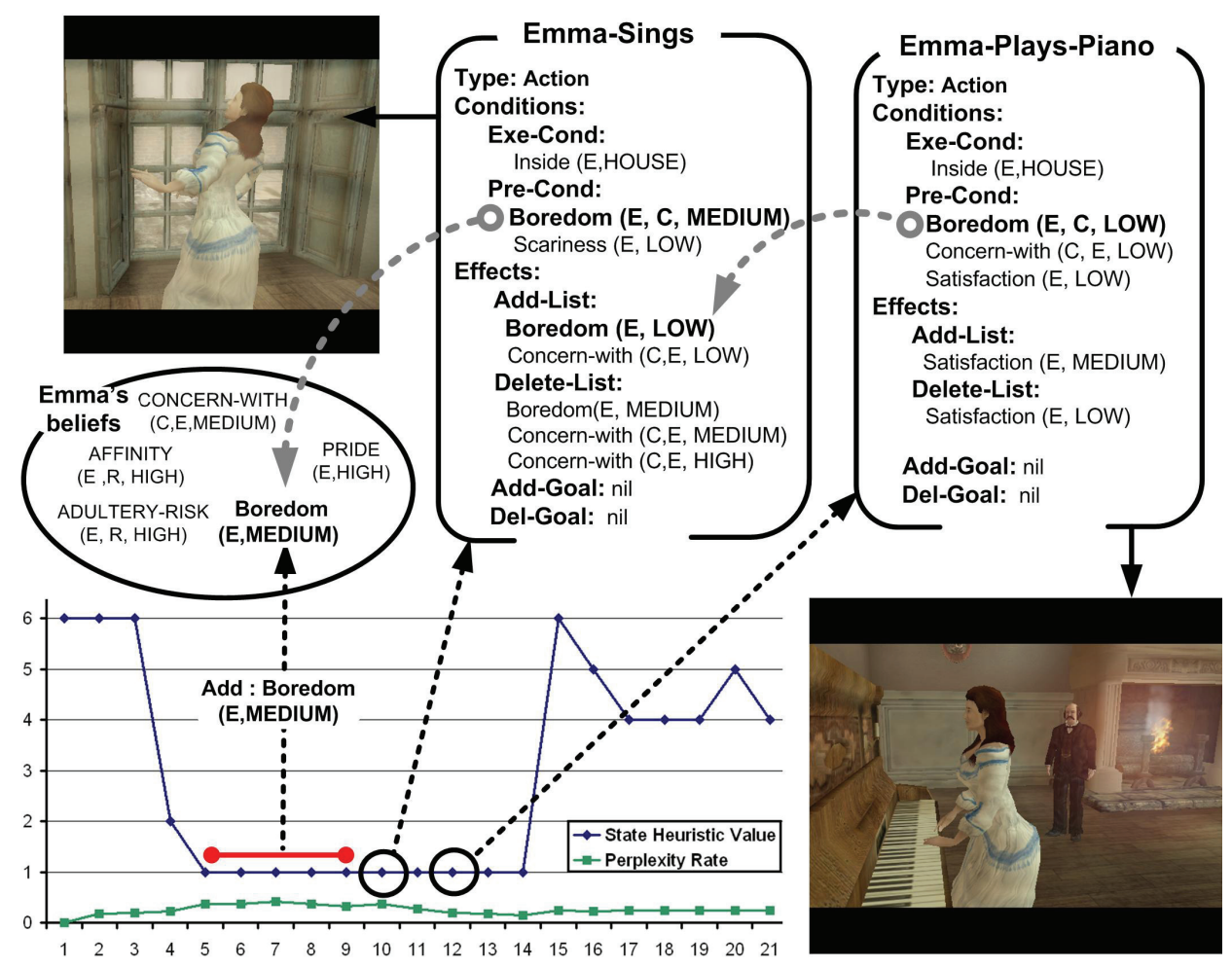

Figure 1: Emotional Feedback from the Awareness of Events' Progression. 
The next step consists in devising knowledge representations supporting the character's awareness of how its own situation evolves as a consequence of its own actions or is affected by other characters. For state-space search, as implemented when using HSP, the contribution of specific actions to an agent's desires is best measured through the heuristic function. This encouraged us to use the variation of the heuristic function as a means to measure the perception by a character of the evolution of its own situation. We are only interested in patterns of variation in the heuristic value. For instance, a monotonic decrease in heuristic function is equivalent to a progressive improvement of the agent situation, as it slowly moves towards the realisation of its desires. A lack of progression in the heuristic value would correspond to an absence of improvement, which could itself be down to lack of opportunities (boredom) or obstacles (e.g. actions from antagonist agents, e.g. Charles' mother in the case of Emma). Figure 1 shows such an example, where the lack of progression generates a feeling of boredom, in turn affecting the choice of Emma's actions. Finally, a step increase in the heuristic value, which persists for several steps, would signal a deterioration of the agent's situation. Should such a step increase follow an initial decrease, this pattern would correspond for instance to the narrative notion of "shattered hopes".

\section{CONCLUSIONS}

Exploring new narrative genres, in which characters' feeling and psychology play a major role, is a challenge for Interactive Storytelling research. We have introduced a narrative approach in which the emphasis is on the use of narrative description of characters' psychology rather than cognitive models. Narrative descriptions of character psychology, such as those provided by the author are also compatible with narrative models of the plot itself [3]. In this approach, planning has been used with a weaker representational stance, without equating the narrative itself to a specific plan instantiation. We have still found that formal aspects of search-based planning, such a heuristic variations and perplexity, could be easily mapped into our representational framework, even if it had been designed from the perspective of narrative content.

\section{ACKNOWLEDGMENTS}

This work has been funded in part by the DTI Technology Programme (Design, Simulation and Modelling) through the BARDS Project, in collaboration with Eidos Interactive plc.

\section{REFERENCES}

[1] Aylett, R., Louchart, S., Dias, J., Paiva, A., and Vala, M. FearNot! - An Experiment in Emergent Narrative. In: T. Panayiotopoulos, J. Gratch, R. Aylett, D. Ballin, P. Olivier and T. Rist (Eds.): Intelligent Virtual Agents, 5th International Working Conference, 2005, pp. 305-316.
[2] Bonet, B. and Geffner. H. Planning as Heuristic Search. Artificial Intelligence Special Issue on Heuristic Search, 129(1), pp. 5-33, 2001.

[3] Bremond, C., Logique du Récit, Paris: Editions du Seuil, 1973 (in French)

[4] Cavazza, M., Charles, F. and Mead, S.J., 2002. Interacting with Virtual Characters in Interactive Storytelling. First ACM Joint Conference on Autonomous Agents and MultiAgent Systems, Bologna, Italy, pp. 318-325.

[5] Charles, F. and Cavazza, M. Exploring the Scalability of Character-based Storytelling. Third ACM Joint Conference on Autonomous Agents and Multi-Agent Systems, New York, USA, 2004, pp. 872-879.

[6] Filkes, R. and Nilsson, N. STRIPS: A New Approach to the Application of Theorem Proving to Problem Solving. Proceedings of the Second International Joint Conference on Artificial Intelligence, 1971, pp. 608-620.

[7] Gratch, J. Emile: Marshalling Passions in Training and Education, in Proceedings of the 4th International Conference on Autonomous Agents, Barcelona, Spain, June 2000 .

[8] Gratch, J. Why you should buy an emotional planner, Proceedings of the Autonomous Agents 1999 Workshop on Emotion-based Agent Architectures (EBAA'99).

[9] Korf, R.E. Real-time heuristic search. Artificial Intelligence, 42:2-3, 1990, pp. 189-211.

[10] Leclerc, Y (Ed.). Plans et Scenarios de Madame Bovary, CNRS Editions, France, 1995 (in French).

[11] Magerko, B., Laird, J.E., Assanie, M., Kerfoot, A. and Stokes, D. AI Characters and Directors for Interactive Computer Games. Proceedings of the Nineteenth National Conference on Artificial Intelligence (AAAI 2004), 2004, pp. $877-883$

[12] Marsella, S. and Gratch, J. A Step Towards Irrationality: Using Emotion to Change Belief, in Proceedings of the First International Joint Conference on Autonomous Agents and Multi-Agent Systems, Bologna, Italy, July 2002

[13] Marsella, S and Gratch, J. Modeling Coping Behaviour in Virtual Humans: Don't Worry, Be Happy. Second International Joint Conference on Autonomous Agents and Multi-Agent Systems (AAMAS 2003), ACM Press, 2003, pp. 313-320

[14] Mateas, M., and Stern, A. A Behavior Language for StoryBased Believable Agents. IEEE Intelligent Systems 17(4), 2002, pp. 39-47.

[15] Riedl, M.O., Young, R.M. An Intent-Driven Planner for Multi-Agent Story Generation. Third ACM Joint Conference on Autonomous Agents and Multi-Agent Systems, New York, USA, 2004, pp. 186-193. 